\title{
A Case Report of Cardiobacterium hominis Endocarditis in a Pregnant Woman
}

\author{
D Sarumathi ${ }^{1}$, G Anitha ${ }^{1}$, Deepashree $\mathrm{R}^{1}$, Rajeev Thilak C ${ }^{2}$, Apurba S. Sastry ${ }^{1}$ \\ 1. Microbiology, Jawaharlal Institute of Postgraduate Medical Education and Research, Puducherry, IND 2. \\ Cardiothoracic Surgery, Jawaharlal Institute of Postgraduate Medical Education and Research, Puducherry, IND
}

Corresponding author: Apurba S. Sastry, drapurbasastry@gmail.com

\begin{abstract}
Infective endocarditis (IE) is an infrequent endovascular disease, which can result in significant mortality and morbidity. Staphylococcus aureus and viridans streptococci remain the most common etiological agent. Cardiobacterium hominis, a member of the HACEK

(Haemophilus species, Aggregatibacter species, Cardiobacterium hominis, Eikenella corrodens, and Kingella species) group of fastidious gram-negative bacillus, is a part of normal upper respiratory flora and a rare cause implicated in IE. Cases of Cardiobacterium hominis endocarditis are being increasingly reported in last few years due to advancement in automated blood culture system such as BacT/ALERT Virtuo $^{\circledast}$ and identification system such as MALDI-TOF MS (matrix-assisted laser desorption/ionization timeof-flight mass spectrometry). We herein report a first case of Cardiobacterium hominis endocarditis in a pregnant woman at 20 weeks of gestation. Following spontaneous abortion and evacuation of the fetus, appropriate surgical intervention under heparinized condition and pathogen-directed medical intervention was initiated in this patient. This case report highlights the importance of appropriate antimicrobial therapy, which augments earlier resolution of the disease.
\end{abstract}

Categories: Cardiac/Thoracic/Vascular Surgery, Internal Medicine, Infectious Disease Keywords: infective endocarditis, cardiobacterium hominis, hacek group, maldi-tof

\section{Introduction}

Infective endocarditis (IE) is a destructive cardiovascular infection associated with high morbidity and mortality $[1,2]$. The most common etiological agent implicated in IE is gram-positive bacteria such as Staphylococcus aureus [1]. The HACEK group of organisms (Haemophilus species, Aggregatibacter species, Cardiobacterium hominis, Eikenella corrodens, and Kingella species) is a rare cause of IE, accounting for approximately $<5 \%$ of IE [3]. Cardiobacterium hominis, a highly fastidious gram-negative bacillus and a normal oral commensal [4], can occasionally cause IE. The identification of $C$. hominis is difficult as it grows very slowly and requires special enriched media for growth [5]. However, with the recent advancement in the diagnostic tools such as the automated blood culture system BacT/ALERT Virtuo ${ }^{\circledR}$ (bioMérieux, Marcy-l'Étoile, France) and matrix-assisted laser desorption/ionization time-of-flight mass spectrometry (MALDI-TOF MS), the cases of C.

Review began $07 / 23 / 2020$ Review began $07 / 23 / 2020$
Review ended 08/07/2020 Published 08/18/2020

\section{() Copyright 2020}

Sarumathi et al. This is an open access article distributed under the terms of the Creative Commons Attribution License CC-BY 4.0., which permits unrestricted use, distribution, and reproduction in any medium, provided the original author and source are credited. hominis endocarditis are being increasingly reported in the recent years [5]. Prompt and accurate diagnosis will help in instituting appropriate management; which includes early surgical intervention along with prolonged antimicrobial therapy [6,7]. In pregnancy, IE is extremely rare, but, when present, it contributes to significant maternal and fetal mortality [8].To the best of our knowledge, no reports of $C$. hominis IE has been reported in pregnant women. We describe a case of mitral valve IE due to C. hominis in a 35-year-old pregnant woman who was successfully treated with medical and surgical interventions.

\section{Case Presentation}

A 35-year-old multigravida woman at 20th week of gestation presented with new onset of chest pain and difficulty in breathing. She had left-sided retrosternal chest pain, which was insidious onset, with on and off episodes, each lasting for one to two minutes with no radiation, aggravated by heavy work and relieved with rest, along with breathlessness, palpitation, pedal edema for one month, and a history of intermittent fever for one-week duration. She did not have other comorbidities, and treatment history was not relevant.

Laboratory examination demonstrated a white blood cell count of 11,000 per microliter and normochromic normocytic anemia (Hb-5gm \%). An electrocardiogram was normal (Figure 1) and a trans-esophageal echocardiography (TEE) (Figure 2) demonstrated $7 \times 12 \mathrm{~mm}$ vegetation attached to the anterior mitral leaflet with chordal rupture, causing severe anterior mitral leaflet prolapse. There was severe eccentric mitral regurgitation (MR) and sub-valvular calcification with mitral stenosis and mild aortic regurgitation. She complained of acute pain in the left lower limb. An arterial Doppler ultrasound was performed, which showed a thrombus in the left femoral artery. Left transfemoral embolectomy was performed. As IE was suspected, the clinical team collected three pairs of blood culture in BacT/ALERT bottles before the start of antibiotics and sent for bacteriological culture. Subsequently, empirical antibiotic therapy was initiated. The 


\section{Cureus}

bottles were immediately loaded in BacT/ALERT Virtuo automated instrument. Meanwhile, pregnancy was spontaneously aborted and fetal products were evacuated. Four out of six blood culture bottles were flagged positive after seven days of the incubation following which gram stain and subculture were performed on to blood agar and Macconkey agar. Gram stain revealed highly pleomorphic gram-negative rods of 1-3 $\mu \mathrm{m}$ in length arranged in short chains and rosettes with an irregular staining pattern. Colonies on blood agar were small, circular, flat, non-hemolytic. Biochemical tests performed from the colonies revealed catalase negative, oxidase positive, indole positive, and nitrate negative. Then the colonies were subjected to MALDI-TOF (version 3.2, bioMérieux), which subsequently was identified as $C$. hominis. The instrument had given a confidence score of $99.9 \%$ for the identification.

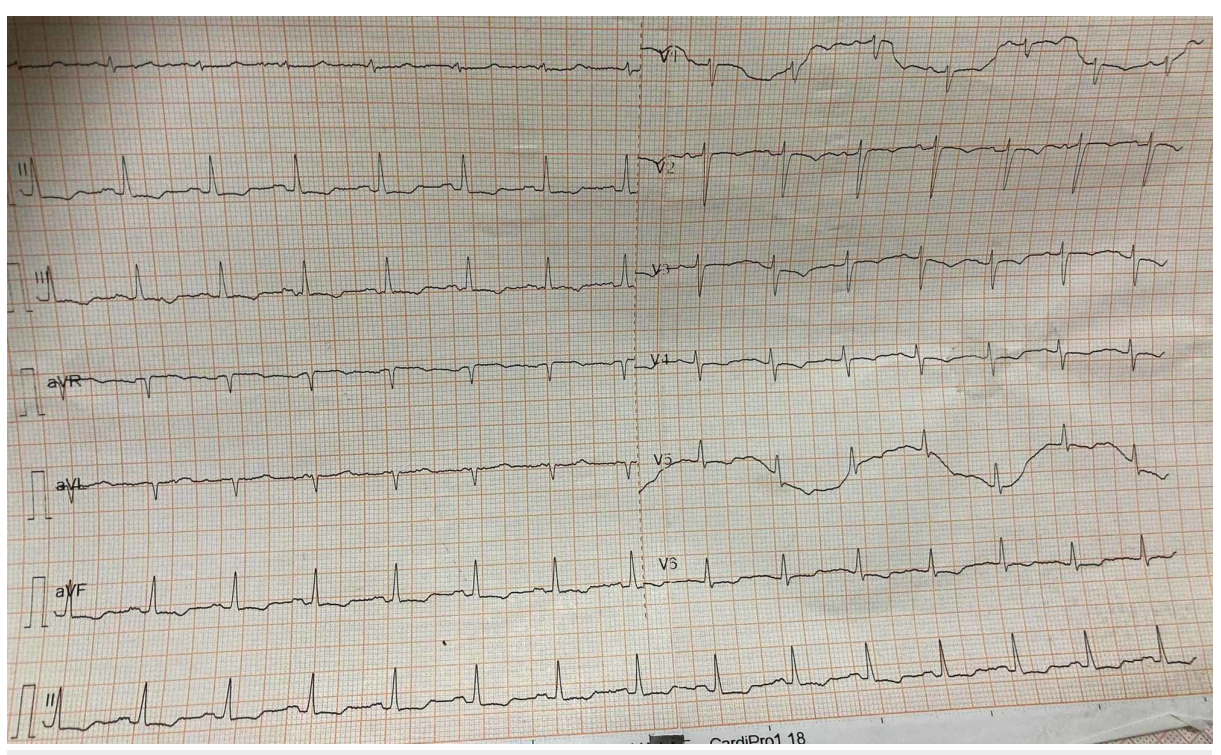

\section{FIGURE 1: Electrocardiogram}

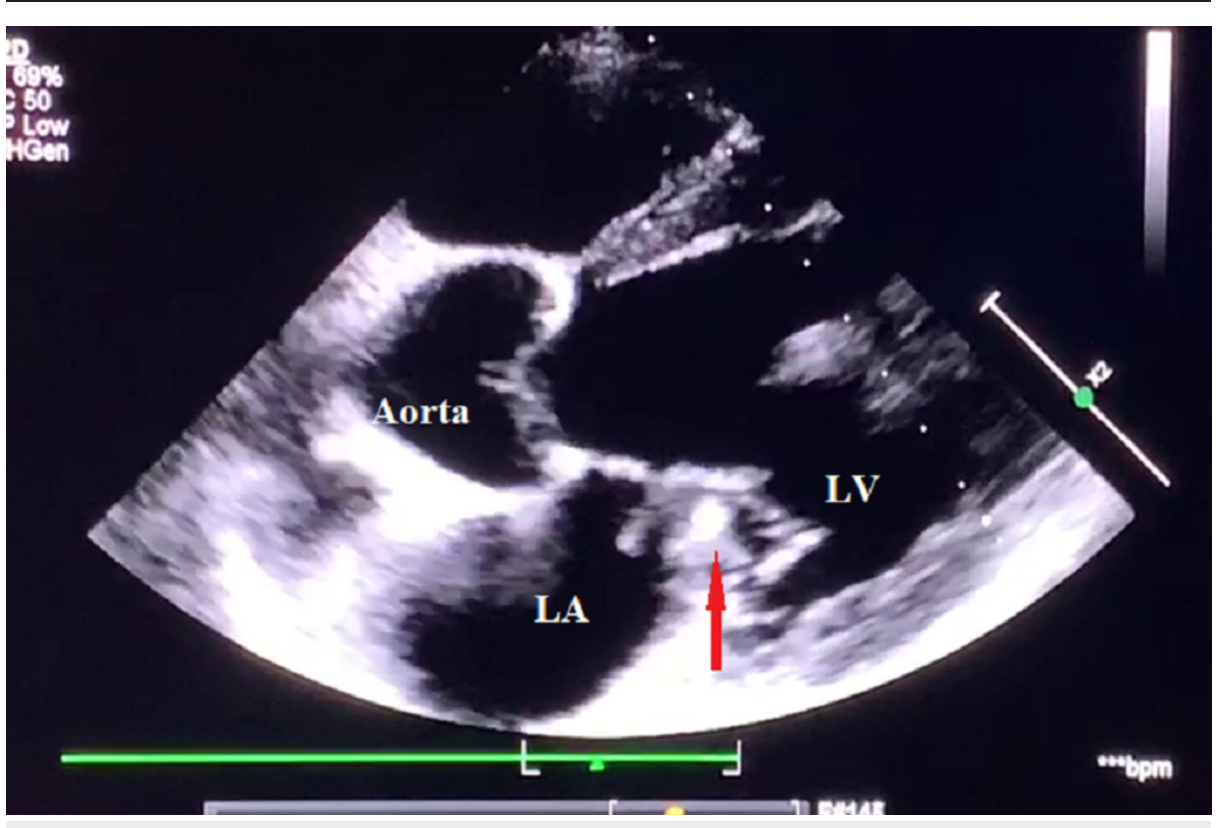

\section{FIGURE 2: Trans-esophageal echocardiography}

Red arrow shows $7 \times 12 \mathrm{~mm}$ vegetation attached to the anterior mitral leaflet.

Based on the culture report targeted therapy comprising of ceftriaxone for four weeks combined with gentamicin for two weeks was initiated. After 14 days of pathogen-directed therapy, the blood culture was repeated and the culture turned out to be sterile. The patient clinically improved and subsequently was discharged. 


\section{Discussion}

IE, a rare but severe form of valvular heart disease, is characterized by inflammatory state of the endocardium and linings of the heart valves [1]. Multiple factors predispose patients to the development of IE, such as intravenous drug use, prosthetic heart valves, structural heart disease, prior rheumatic fever, recent dental procedure, previous endocarditis, and congenital valvular disease [9]. The diagnosis of IE is based on the modified Duke's criteria, which incorporate information from echocardiography, history and physical examination, blood culture, and histopathology [10]. The pathogenesis involves endothelial injury triggering sterile thrombus and subsequent adhesion of bacteria, which together constitute mature vegetation. Such bacteria enter through a traumatized surface mucosa of the body, probably the oral cavity, gut, or genitourinary tract [1].

Various studies have shown Staphylococcus aureus as the leading organism involved in native valve endocarditis (NVE) followed by viridans streptococci, Enterococcus, and coagulase-negative staphylococci [1,3]. In more than $10 \%$ of IE, called as culture-negative IE, the causative agent is unknown. The classical example is the HACEK group of organisms. However, the word "culture-negative IE" is a misnomer as with the advent of sophistically automated technique it is now possible to isolate many of these fastidious organisms including HACEK. BacT/ALERT Virtuo is an advanced automated culture method that works on the same principle of BacT/ALERT. However, it offers several advantages such as automated loading and unloading of bottles without opening the doors, which helps to provide optimal incubatory condition without temperature fluctuations.

Cardiobacterium hominis is one among the HACEK group and is an uncommon cause of IE. It is a fastidious gram-negative coccobacillus that belongs to the family Coriobacteriaceae (includes three genera: Cardiobacterium, Dichelobacter, and Suttonella) [11]. Cardiobacterium hominis is considered as microaerophilic, which grows best in a humid atmosphere with an increased

$\mathrm{CO}_{2}$ tension [12]. Cardiobacterium hominis is considered as a member of the normal flora of the mouth and has been recovered from the nose or throat of normal individuals [4]. It rarely causes human infections such as endovascular infections, septic arthritis, ocular infections, and neonatal sepsis. It generally follows a subacute course of IE [13]. It is of low virulence and affects previously damaged or prosthetic valves [9]. It has a strong association with aortic valve infection and is known to produce large friable vegetations [3]. Penicillin along with aminoglycosides was the standard treatment regimen given for HACEK endocarditis in the past. It is found that $C$. hominis is generally susceptible to penicillin, ampicillin, cephalosporins, fluoroquinolones, chloramphenicol, and tetracycline. Beta lactamase producing strains of Cardiobacterium have been described recently [9]. The American Heart Association/Infectious Disease Society of America endocarditis guidelines recommend monotherapy regimen comprising either ceftriaxone ( $2 \mathrm{~g} / 24$ hours IV/IM in one dose) or ampicillin-sulbactam for a duration of four weeks for HACEK endocarditis [14].

\section{Conclusions}

The case report highlights the significance of a rare pathogenic agent $C$. hominis responsible for IE in a pregnant woman of 20 weeks' gestational age. We also emphasize the importance of automated blood culture systems, especially BacT/alert Virtuo and better pathogen identification methods such as MALDITOF MS, which aid in the appropriate antimicrobial therapy, thereby augmenting the earlier resolution of the disease.

\section{Additional Information \\ Disclosures}

Human subjects: Consent was obtained by all participants in this study. Conflicts of interest: In compliance with the ICMJE uniform disclosure form, all authors declare the following: Payment/services info: All authors have declared that no financial support was received from any organization for the submitted work. Financial relationships: All authors have declared that they have no financial relationships at present or within the previous three years with any organizations that might have an interest in the submitted work. Other relationships: All authors have declared that there are no other relationships or activities that could appear to have influenced the submitted work.

\section{References}

1. McDonald JR: Acute infective endocarditis . Infect Dis Clin North Am. 2009, 23:643-664. 10.1016/i.idc.2009.04.013

2. Holland TL, Baddour LM, Bayer AS, Hoen B, Miro JM, Fowler VG Jr: Infective endocarditis. Nat Rev Dis Primers. 2016, 2:16059.

3. Chambers ST, Murdoch D, Morris A, et al.: HACEK infective endocarditis: characteristics and outcomes from a large, multi-national cohort. PLoS One. 2013, 17:63181. 10.1371/journal.pone.0063181

4. Malani AN, Aronoff DM, Bradley SF, Kauffman CA: Cardiobacterium hominis endocarditis: two cases and a review of the literature. Eur J Clin Microbiol Infect Dis. 2006, 25:587-595. 10.1007/s10096-006-0189-9

5. Asai N, Sakanashi D, Suematsu H, et al.: Infective endocarditis caused by Cardiobacterium hominis endocarditis: a case report and review of the literature. J Infect Chemother. 2019, 25:626-629. 10.1016/j.jiac.2019.02.002 
6. Horstkotte D, Follath F, Gutschik E, et al.: Guidelines on prevention, diagnosis and treatment of infective endocarditis executive summary: the Task Force on Infective Endocarditis of the European Society of Cardiology. Eur Heart J. 2004, 25:267-276. 10.1016/j.ehj.2003.11.008

7. Kang DH, Kim YJ, Kim SH, et al.: Early surgery versus conventional treatment for infective endocarditis . N Engl J Med. 2012, 28:2466-2473. 10.1056/NEJMoa1112843

8. Connolly C, O’Donoghue K, Doran H, McCarthy FP: Infective endocarditis in pregnancy: case report and review of the literature. Obstet Med. 2015, 8:102-104. 10.1177/1753495X15572857

9. Walkty A: Cardiobacterium hominis endocarditis: a case report and review of the literature . Can J Infect Dis Med Microbiol. 2005, 16:293-297. 10.1155/2005/716873

10. Li JS, Sexton DJ, Mick N , et al.: Proposed modifications to the Duke criteria for the diagnosis of infective endocarditis. Clin Infect Dis. 2000, 30:633-638. 10.1086/313753

11. Moore ER, Mihaylova SA, Gomila M: The family Cardiobacteriaceae. The Prokaryotes: Gammaproteobacteria. Rosenberg E, DeLong EF, Lory S, Stackebrandt E, Thompson F (ed): Springer, Berlin/Heidelberg; 2014. 135-146. 10.1007/978-3-642-38922-1

12. Wormser GP, Bottone EJ: Cardiobacterium hominis: review of microbiologic and clinical features . Rev Infect Dis. 1983, 5:680-691. 10.1093/clinids/5.4.680

13. Vasanthamohan L, Kronfli N, Hou C, Prebtani AP, Shroff A: Subacute infective endocarditis secondary to cardiobacterium hominis in a patient with mitral valve prolapse. Can J Gen Intern Med. 2017, 12:1-4. 10.22374/cjgim.v12i3.161

14. Baddour LM, Wilson WR, Bayer AS, et al.: Infective endocarditis. Diagnosis, antimicrobial therapy, and management of complications: a statement for healthcare professionals from the Committee on Rheumatic Fever, Endocarditis, and Kawasaki Disease, Council on Cardiovascular Disease in the Young, and the Councils on Clinical Cardiology, Stroke, and Cardiovascular Surgery and Anesthesia, American Heart Association. Circulation. 2005, 14:394-434. 10.1161/CIRCULATIONAHA.105.165564 